\title{
Intensive care medicine comes of age
}

\author{
Julian Bion and Timothy Evans
}

On 1 August 2012, the Faculty of Intensive Care Medicine $(\text { FICM })^{1}$ will welcome the first trainees to be appointed to the UK's new primary specialty training programme for intensive care medicine (ICM). The foundation of the faculty and the establishment of the new programme represent the culmination of the efforts of many individuals and organisations over the past 40 years. Both a destination and a new beginning, it is worth considering the pathways which led to this point, and how we should plot our future course in partnership with the seven trustee colleges which have supported our growth, in particular the Royal College of Anaesthetists and the Royal College of Physicians, and our sister organisation the Intensive Care Society.

The polio epidemics of the 1950s were the stimulus for colocating patients requiring ventilatory support. Combined with manual positive pressure ventilation through a tracheostomy, blood gas measurement and intensive nursing care, the mortality of these patients was reduced from nearly $90 \%$ to $40 \%$. $^{2,3}$ With the eradication of polio through effective immunisation, the new technique of mechanical ventilation came to be applied to prolonged support of all critically ill patients with multiple organ failure, including those with adult respiratory distress syndrome (ARDS), ${ }^{4}$ and particularly in the setting of sepsis. Sixty years on, there is a more sophisticated understanding of the pathogenesis of critical illness and sepsis, but few specific effective therapies. ${ }^{5}$ The capacity to support most organ systems exists but patient outcomes are improved by adopting strategies to minimise harm caused by these forms of support. ${ }^{6-8}$ The benefits of early intervention and early rehabilitation have also been recognised, but the limitations of intensive care, particularly in the setting of chronic illness and physical dependence, have become increasingly apparent. We work in a complex, pressured, highly monitored and data-rich environment, but appreciate the need to integrate and improve reliable delivery of best practice care inside as well as outside the intensive care unit. ${ }^{9}$ The specialists who work in this environment must therefore combine the capacities of the family doctor, acute physician, physiologist, technician, palliative care practitioner, researcher, ethicist, team leader and human factors expert. This skill set makes interesting demands of specialist training programmes. Until recently there was substantial diversity in content, duration and ownership of ICM worldwide, with no clear 'product specification' for an intensive care specialist. This is now changing through initiatives such as the international programme Competency-based

Julian Bion, dean; Timothy Evans, vice-dean

Faculty of Intensive Care Medicine training in intensive care in Europe (CoBaTrICE), ${ }^{10,11}$ which has developed core skills across the three models of ICM training worldwide: as a sub-specialty of one or more disciplines; as a 'supra-specialty' (an add-on with common skills across all), and primary specialty ICM. There is considerable overlap in content between the supra-specialty and primary specialty models, reflecting the desire among intensive care specialists to retain the multidisciplinary nature of ICM while developing the status and professional confidence of a primary specialty denoted by the award of a certificate of completion of specialist training (CCT). In the UK this approach has been formalised by allowing trainees to follow the seven-year primary specialty (single CCT) programme alone, or to undertake the ICM CCT programme in conjunction with another primary specialty (dual CCT). Depending on the partner specialty, dual CCT will take 18-36 months longer than a single CCT by offsetting or sharing common competencies between programmes.

There are several technical challenges associated with implementing these two models of training, so we will start the process by appointing to a relatively small number of new training posts while permitting entry for a further year (until 31 July 2013) to the current joint CCT programme. Agreement has been secured from the General Medical Council (GMC) for 'stepped appointment' to dual CCT programmes, allowing trainees up to 18 months to secure a place on their desired partner specialty programme following appointment to ICM or to ICM in the event of being appointed first to a non-ICM programme. The GMC will use the experience gained from ICM to develop dual programmes in other disciplines.

The support of training programme directors in partner specialties will be needed to ensure satisfactory integration of ICM with training in the complementary elements of medicine and anaesthesia. A longer-term ambition is to wholly-own all ICM training posts instead of part-borrowing training time from other specialties. The need to increase the number of training posts is also essential, partly to compensate for the longer training time required by the new programme but also to respond to the likely increase in demand for specialists as intensive care services grow. The faculty is therefore working with trusts and deaneries, to approve for training the many noncareer posts currently fully funded for service provision in intensive care, and with the Centre for Workforce Intelligence, to develop robust models which will take into account future service needs while ensuring that ICM becomes an increasingly attractive long-term career.

On the basis that better doctors mean better patient care, the faculty is also developing a strategy which responds to the challenge from Sir John Tooke to move medical education from competence 
to excellence. ${ }^{12}$ Key components include life-long reflective learning, research and audit, the development of evidence-based standards of care, and transdisciplinary teamworking, integrated within a shared framework entitled the Quality Improvement Forum (QIF). The first component involves incorporating techniques for self-awareness and reflection, such as structured observation, multisource feedback, and appraisal and revalidation, within a new continuum of undergraduate, postgraduate and specialist education in ICM. Research and audit represent a spectrum of enquiry-based activities; all trainees and specialists should participate in audit, but we need to make space within the curriculum for research training, and to increase opportunities for trainees to gain earlystage academic clinical fellowships for those wishing to develop their research capacities. Standards will be developed within the framework set by the Department of Health. ${ }^{13}$ Transdisciplinary teamworking is based in part on shared competencies, already achieved for advanced critical care practitioners, ${ }^{14}$ but also suitable for intensive care nurses and other professional groups integral to the care of critically ill patients. Supported conceptually by many partner organisations and by the Department of Health, the QIF will enable the faculty to incorporate new knowledge rapidly within postgraduate training and professional development programmes, to develop or disseminate tools which enhance implementation of best practice at the bedside, and to collaborate in human factors research focused on improving the reliability of care of critically ill patients and their families.

\section{References}

1 Faculty of Intensive Care Medicine. www.ficm.ac.uk

2 Lassen HCA. A preliminary report on the 1952 epidemic of poliomyelitis in Copenhagen with special reference to the treatment of acute respiratory insufficiency. Lancet 1952;ii:1168-75.
3 Ibsen B. The anæsthetist's viewpoint on the treatment of respiratory complications in poliomyelitis during the epidemic in Copenhagen, 1952. Proc R Soc Med 1954;47:72-4.

4 Ashbaugh DG, Bigelow DB, Petty TL, Levine BE. Acute respiratory distress in adults. Lancet 1967;ii:319-23.

5 Sweeney DA, Danner RL, Eichacker PQ, Natanson C. Once is not enough: clinical trials in sepsis. Int Care Med 2008;34:1955-60.

6 The Acute Respiratory Distress Syndrome Network. Ventilation with lower tidal volumes as compared with traditional tidal volumes for acute lung injury and the acute respiratory distress syndrome. $N$ Engl J Med 2000;342:1301-8.

7 Strøm T, Martinussen T, Toft P. A protocol of no sedation for critically ill patients receiving mechanical ventilation: a randomised trial. Lancet 2010;375:475-80.

8 de Smet AM, Kluytmans JA, Cooper BS et al. Decontamination of the digestive tract and oropharynx in ICU patients. $N$ Engl J Med 2009;360:20-31.

9 Department of Health. Comprehensive critical care: a review of adult critical care services. London: DH, 2000.

10 Competency-based training in intensive care in Europe. www.cobatrice.org

11 The CoBaTrICE Collaboration. Development of core competencies for an international training programme in intensive care medicine. Int Care Med 2006;32:1371-83.

12 Tooke J. Aspiring to excellence: final report of the independent enquiry into Modernising Medical Careers. London: MMC, 2008.

13 Quality accounts 2010/11. www.dh.gov.uk/en/Healthcare/ Qualityandproductivity/Makingqualityhappen/qualityaccounts/ index.htm

14 Department of Health. The national education and competence framework for advanced critical care practitioners. London: DH, 2008. www.dh.gov.uk/en/Publicationsandstatistics/Publications/Publications PolicyAndGuidance/DH_084011

Address for correspondence: Professor J Bion, Faculty of Intensive Care Medicine, Churchill House, 35 Red Lion Square, London WC1R 4SG. Email: ficm@rcoa.ac.uk 